\title{
Sensory Loss Mimicking Cauda Equina Syndrome due to Cervical Spinal Lesion in a Patient with Clinically Isolated Syndrome
}

\author{
Giulia Vinceti Andrea Zini Paolo Nichelli \\ Jessica Mandrioli \\ Department of Neuroscience, University of Modena and Reggio Emilia, \\ S. Agostino-Estense Hospital, Modena, Italy
}

\section{Key Words}

Polyradiculopathy · Cauda equina syndrome $\cdot$ Magnetic resonance imaging $\cdot$ Spinal diseases

\begin{abstract}
We describe the case of a 39-year-old woman with signs and symptoms suggesting cauda equina syndrome. Lumbosacral magnetic resonance imaging (MRI) demonstrated no lesion at this level, while cervical MRI showed a T2-hyperintense lesion in the middle-right anterolateral region of the cervical spinal cord, which may explain the symptoms by involving the anterior spinothalamic tract. We suggest that in cases with cauda equina syndrome presentation and normal lumbosacral MRI, a cervicodorsal lesion should be considered during diagnostic assessment.
\end{abstract}

\section{Introduction}

Cauda equina syndrome (CES) is a complex clinical entity due to compression of lumbosacral spinal nerve roots encompassed in the dural sac. The fully developed syndrome is characterized by multiple sensory signs and symptoms, including low back pain, saddle hypoesthesia or anesthesia, weakness of the lower extremities and impaired low reflexes, as well as sphincter dysfunction and sexual impotence [1]. The clinical picture of CES may be asymmetric and can substantially differ in each patient, with various combinations of signs and symptoms, often presenting as partial disease onset. CES etiology includes compressive causes, either structural or neoplastic, but 
also non-compressive causes (ischemic, inflammatory or infectious) [1, 2]. Among compressive causes, lumbosacral disc prolapse is predominant in frequency compared to spinal neoplasms and spinal stenosis, accounting for 45,29 , and $21 \%$ of all cases, respectively [2]. Despite the wide range of these causative conditions, the majority of them share a common localization at the lumbosacral level. We present the case of a woman with a clinical picture suggesting CES whose etiology could be assigned to a cervical spinal cord lesion.

\section{Case Report}

A 39-year-old woman presented with a three-day history of paresthesias in the saddle region, posterior thighs, legs and soles, mainly on the left side. She also complained of sensory loss at the perineal level, but denied urinary retention or urgency. Neurological examination showed only mild hypoesthesia in the perianal and perigenital regions, especially on the left. Lumbosacral magnetic resonance imaging (MRI) was performed, revealing no lesion at this level (fig. 1). Pelvis CT scans were also normal. The electrophysiological assessment showed delayed conduction of the somatosensorial pathway at somatosensory evoked potentials for stimulus applied to the left leg. Routine laboratory tests, including inflammation indexes, autoimmune and neoplastic laboratory assays, were within the normal range. Cerebrospinal fluid (CSF) examination revealed a cell count of 13 cells $/ \mathrm{mm}^{3}$, mainly lymphocytes, while CSF total protein content was normal. Abundant oligoclonal IgG bands and intrathecal IgG synthesis were also detected. Blood-brain barrier damage was absent. Serum and CSF antibodies to Borrelia burgdorferi and Treponema pallidum were negative, as were CSF culture for bacteria and fungi and PCR assays for an extensive virus panel. Cervical MRI showed a T2-hyperintense lesion in the middle-right anterolateral region of the cervical (C2) spinal cord (fig. 2a, b). Subcortical lesions were also present. This episode was considered a clinically isolated syndrome suggestive of multiple sclerosis, and a high dose of intravenous methylprednisolone was administered. Complete clinical recovery followed, and one month later brain and cervical MRI revealed a considerable improvement in cervical MRI with size reduction of the previously detected lesion. One year later no relapses occurred; no new lesions were detected at neuroimaging during follow-up.

\section{Discussion}

As previously mentioned, all potential organic explanations for a clinical picture suggestive of CES are typically searched for within the lumbosacral portion of the spine, and lumbar spine MRI is currently the method of choice to visualize and investigate cauda equina nerve roots within the thecal sac, as suggested by the limited available literature which identified lumbosacral MRI scans as gold standard for CES diagnosis [3-5]. However, in some cases CES patients may have normal lumbosacral MRI (around $50 \%$ of cases), as well as no other clear organic causes of CES, leading to an assumption of a 'functional' origin of the clinical findings [4]. The present observations suggest the possibility to consider a cervicodorsal lesion during diagnostic assessment in cases of CES with normal lumbosacral MRI, variability of spinal cord lesion localization being an important point to consider given the somatotopic organization of the spinothalamic sensory fibers (fig. 2c). Indeed, we cannot rule out that the lesion found in this particular case is an incidental finding, but the MRI improvement detected one month later points to a temporal relation between the symptoms and the cervical lesion, which in our case may actually explain the clinical picture mimicking CES. Moreover, bowel, bladder and sexual dysfuntion, which are part of the fully developed CES, were missing in our case, as expected since the relevant afferent and efferent neural circuits are 
located at the lumbosacral level of the spine. In conclusion, we suggest that in cases presenting with CES symptoms and normal lumbosacral MRI, a cervicodorsal lesion should be considered during diagnostic assessment.

\section{Disclosure Statement}

The authors have no conflicts of interest to disclose.

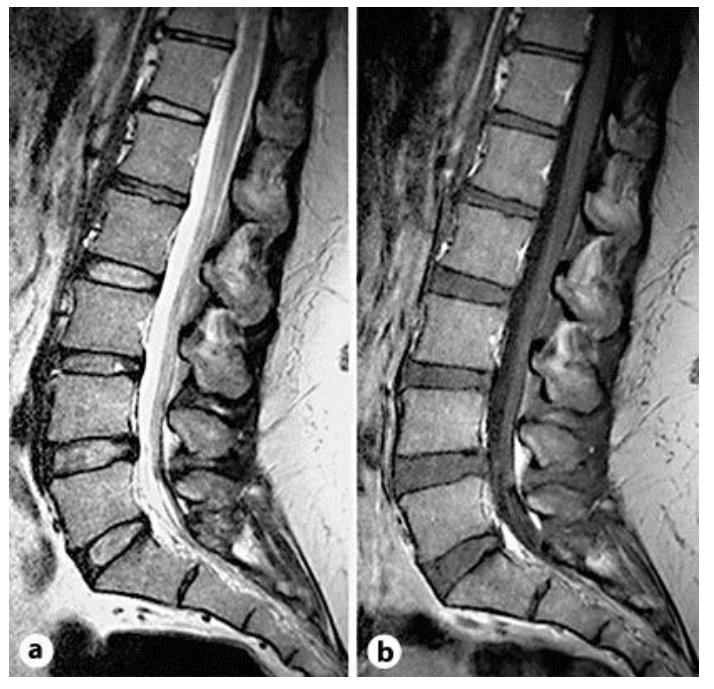

Fig. 1. Sagittal T2-weighted (a) and post-gadolinium T1-weighted (b) MRI showing no lesions in the lumbosacral region.
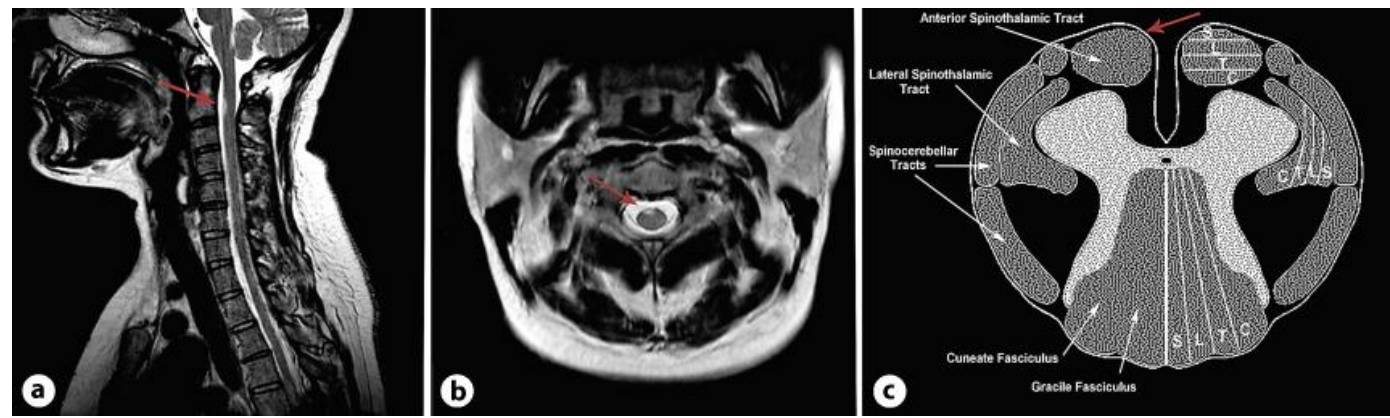

Fig. 2. Sagittal (a) and axial (b) T2-weighted MRI: cervical lesion (C2) in the middle-right anterolateral region. c Somatotopic organization of the anterior spinothalamic tract: sacral sensory projections (S) localize anteriorly to lumbar (L), thoracic (T) and cervical (C) ones, which join posteriorly. 


\section{References}

-1 Orendácová J, Cízková D, Kafka J, Lukácová N, Marsala M, Sulla I, Marsala J, Katsube N: Cauda equina syndrome. Prog Neurobiol 2001;64:613-637.

-2 Fraser S, Roberts L, Murphy E: Cauda equina syndrome: a literature review of its definition and clinical presentation. Arch Phys Med Rehabil 2009;90:1964-1968.

3 Bell DA, Collie D, Statham PF: Cauda equina syndrome: what is the correlation between clinical assessment and MRI scanning? Br J Neurosurg 2007;21:201-203.

4 Rooney A, Statham PF, Stone J: Cauda equina syndrome with normal MR imaging. J Neurol 2009;256:721-725.

5 Balasubramanian K, Kalsi P, Greenough CG, Kuskoor Seetharam MP: Reliability of clinical assessment in diagnosing cauda equina syndrome. Br J Neurosurg 2010;24:383-386. 